\title{
Endoscopic Retrieval of a Proximally Migrated Biliary Plastic Stent Using Direct per-Oral Cholangioscopy
}

\author{
Roberta Maselli ${ }^{1}$, Edoardo Troncone ${ }^{2}$, Alessandro Fugazza ${ }^{1}$, Francesco Auriemma ${ }^{3}$, Andrea Anderloni ${ }^{1}$, Annalisa \\ Cappello $^{1}$, Alessandro Repici ${ }^{1,4}$
}

1) Digestive Endoscopy Unit, Division of Gastroenterology, Humanitas Research Hospital, Rozzano, Milan; 2) Department of Systems Medicine, University of Rome Tor Vergata, Rome; 3) Gastrointestinal Endoscopy Unit, Humanitas - Mater Domini, Castellanza, Varese; 4) Humanitas University, Rozzano, Milan, Italy
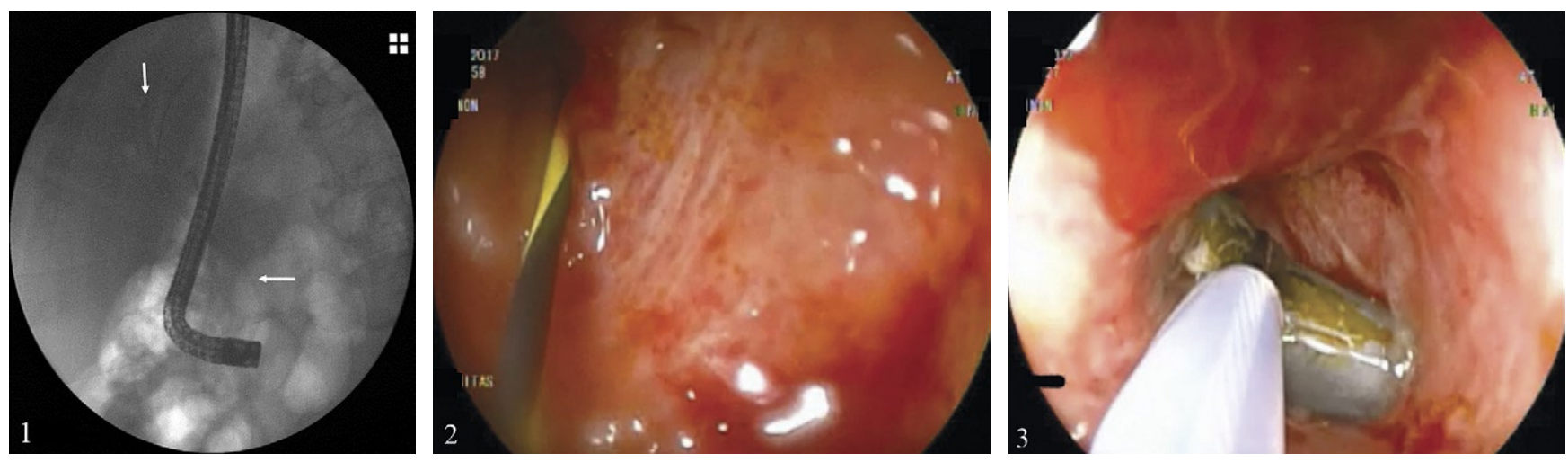

A 61 year old male with jaundice and suspected Mirizzi syndrome underwent an endoscopic retrograde cholangiopancreatography with a placement of a $9 \mathrm{~cm}-10$ French biliary plastic stent and then underwent an uneventful cholecystectomy. After surgery, an ERCP was attempted to remove the plastic stent, but the distal part of the stent was no longer visible in the second part of the duodenum. Fluoroscopy image confirmed the proximal migration of the stent into the common bile duct (CBD) (Fig. 1). Several retrieval attempts using Dormia basket, foreign body grasping forceps, balloon catheters and a polypectomy snare were performed without success. Therefore, after an endoscopic papillary large balloon dilation up to $12 \mathrm{~mm}$, a direct per-oral cholangioscopy (DPOC) using a $8.5 \mathrm{~mm}$ diameter slim endoscope (FujiFilm EG 530FP) was performed by the per-oral route, with a 0.035 inch guidewire assistance for intubating the papilla. Direct per-oral cholangioscopy showed the migrated stent into the medium CBD (Fig. 2), which was successfully retrieved using a $15 \mathrm{~mm}$ snare (Captivator II; Boston Scientific Corp., Marlborough, Massachusetts, USA) through the $2.8 \mathrm{~mm}$ working channel of the endoscope under direct visualization (Fig. 3). No adverse events during the procedure or in the subsequent follow-up were reported.

Migration of plastic biliary stent occurs in $5-10 \%$ of cases and endoscopic management represents the first-line therapeutic approach $[1,2]$. However, endoscopic retrieval can be challenging and more invasive procedures (i.e. radiological interventions, surgery) may be required in some difficult cases. Direct per-oral cholangioscopy permits the performing of diagnostic and therapeutic endoscopic procedures in the biliary tract under direct visualization [3]. In the presented case, we reported a new therapeutic approach using DPOC, for possible challenges in the case of a proximally migrated biliary plastic stent, avoiding the need of further invasive procedures for the patient.

Corresponding author: Roberta Maselli, roberta.maselli@humanitas.it

Conflicts of interest: None to declare.

\section{REFERENCES}

1. Chaurasia OP, Rauws EA, Fockens P, Huibregtse K. Endoscopic techniques for retrieval of proximally migrated biliary stents: the Amsterdam experience. Gastrointest Endosc 1999;50:780-785. doi:10.1016/S0016-5107(99)70158-6

2. Arhan M, Odemis B, Parlak E, Ertugrul I, Basar O. Migration of biliary plastic stents: experience of a tertiary center. Surg Endosc 2009;23:769775. doi:10.1007/s00464-008-0067-x

3. Anderloni A, Fugazza A, Auriemma F, et al. Intrabiliary resection of metastasis originating from colorectal carcinoma during direct peroral cholangioscopy: a new tool for biliary palliation. Endoscopy 2018;50:E97-E98. doi:10.1055/s-0044-100487 